\section{An unusual case of laryngeal mask airway obstruction}

To the Editor:

A healthy 46-yr-old woman was booked for bunion surgery under general anesthesia. After the iv induction of anesthesia, a \#4 laryngeal mask airway (LMA) was inserted uneventfully but ventilation was impossible. The LMA was removed and the patient was ventilated easily by facemask. The LMA was noted to have a foreign object in the lumen. Another LMA was inserted and the patient was ventilated without difficulty. No desaturation occurred throughout the incident. The patient made an uneventful recovery.

The LMA was totally obstructed by a cylindrical plastic plug, which we were able to push out the mask end with a stylet (Figure).

A visit to our sterilization department revealed the cause. The object was a plastic cap on the stainless steel posts of the "Utility Rack Insert" (Getinge, Castle corporation). The sterilizing process had recently been changed to mount the LMA vertically; connector end first, onto the plastic capped posts before the wash cycle. When finished the mask was removed - apparently taking the cap with it. It was then packaged in a sterile bag with a transparent window for steam autoclaving. After the incident, the caps were easily removed from the posts and discarded. Sterilizing techniques were changed to prevent a recurrence.

A closer inspection of the LMA would have detected the plug but, if situated near either end, this would be less obvious. It could have been disastrous for the

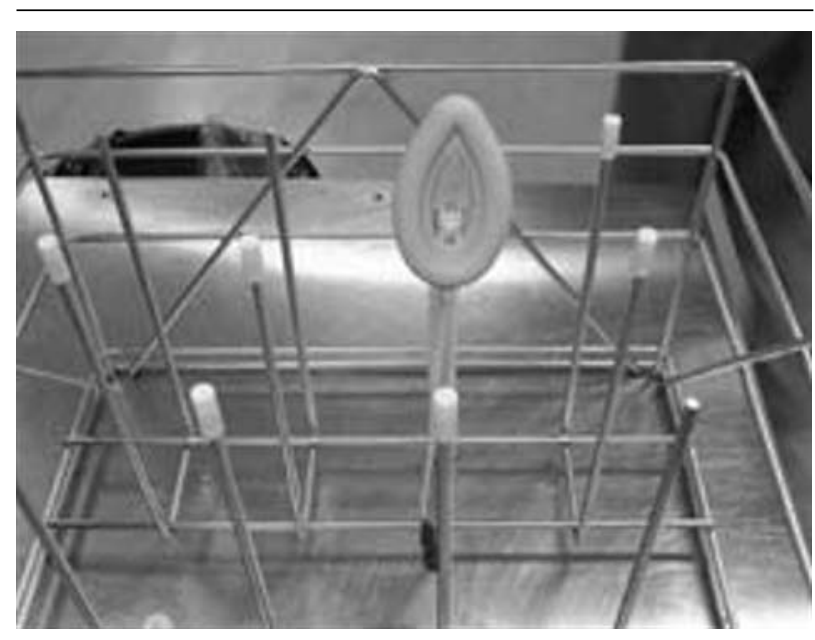

FIGURE Laryngeal mask airway (LMA) mounted on the stainless steel post of the "Utility Rack Insert" prior to sterilization. patient if the object had been forced out through the LMA bars into the patient's airway.

There are other reports of foreign objects in LMA's after sterilization ${ }^{1-3}$ including one where an entire steel cleaning post was found in the LMA. ${ }^{4}$

This report highlights the danger of inventing new processes or using a tool for a purpose it was not intended and the importance of a "pre-flight" inspection of all airway equipment.

Douglas Hardy MD FRCPC

Ottawa, Ontario

\section{References}

1 Conacher ID. Foreign body in a laryngeal mask airway (Letter). Anaesthesia 1991; 46: 164.

2 Cameron D, Onslow J. Obstruction of airway equipment (Letter). Anaesthesia 2002; 57: 188-9.

3 Riley RH, Browning FS. Another foreign body in a laryngeal mask airway (Letter). Anaesthesia 1996; 5: 286-7.

4 Srikanth $K$. Yet another foreign body in a laryngeal mask airway (Letter). Anaesthesia 2002; 57: 189-90.

\section{Successes and failures with the laryn- geal mask airway (LMA) in patients with Treacher Collins syndrome - a}

\section{case series}

To the Editor:

Treacher Collins syndrome (TCS) is a disease known to be associated with difficult airway. ${ }^{1,2}$ Some authors reported that the laryngeal mask airway (LMA) enabled ventilation in TCS patients. ${ }^{3,4}$ However, it remains unclear whether or not the LMA always provides a patent airway in TCS patients. In the present study, clinical and fibreoptic assessment of the positioning of the LMA was performed in TCS patients. After obtaining institutional approval and informed consent, we studied seven consecutive TCS patients scheduled for elective surgery. Anesthesia was induced with propofol, sevoflurane, and/or fentanyl. No muscle relaxants were used. The LMA was inserted in a standard fashion. In all patients, the LMA was inserted into the hypopharynx on the first attempt. When manual ventilation via the LMA produced sufficient tidal volume $(>8$ $\left.\mathrm{mL} \cdot \mathrm{kg}^{-1}\right)$, it was considered an acceptable patent airway. The pressure at which an audible leak occurred was documented. The position of the LMA was ascertained with a fibrescope, and the view was graded as follows: grade 1 , only larynx seen; grade 2 , epiglottis and larynx 